\title{
Spectral efficient compressive transmission framework for wireless communication systems
}

\author{
Shree Krishna Sharma ${ }^{1}$, Mohammad Patwary ${ }^{2}$, Mohamed Abdel-Maguid ${ }^{3}$ \\ ${ }^{1}$ Interdisciplinary Centre for Security-Reliability and Trust (SnT), University of Luxembourg, L-2721 Luxembourg \\ ${ }^{2}$ Faculty of Computing, Engineering and Sciences, Staffordshire University, Stafford, ST18 OAD, UK \\ ${ }^{3}$ School of Science, Technology and Health, University Campus Suffolk, Ipswich, IP4 10J, UK \\ E-mail: shree.sharma@uni.lu
}

\begin{abstract}
Increasing demand of high-speed data rate is leading to a challenging task to provide services to the users within exponentially growing market for wireless multimedia services. Subsequently, the available radio resources are becoming scarce because of different factors such as spectrum segmentation and dedicated frequency allocation to existing wireless standards. Exploring new techniques for enhancing the spectral efficiency in wireless communication has been an important research challenge. In this study, the enhancement of spectral efficiency of wireless communication systems is considered. A framework is proposed to implement the concept of compressive sampling (CS) for compressing the natural random signals. The performance of proposed framework is evaluated in the context of multiple input multiple output orthogonal frequency division multiplexing system. Simulation-based results show that $25 \%$ of resources can be saved by marginal trade-off with the quality of service (QoS) requirement applying CS to the natural random signals. Furthermore, it can be claimed that this QoS trade-off can be optimised with dynamic selection of random measurement matrices.
\end{abstract}

\section{Introduction}

The rapid development of wireless networks and technologies has caused the appearance of plenty of wireless and mobile devices as well as applications, that attracts more users to demand more radio resources from the network. The spectrum resource is scarce and it is becoming congested because of new applications emerging day by day. Exploring efficient techniques for enhancing spectrum efficiency assuring the satisfied quality of service (QoS) has become an open research challenge.

Recently, compressive sampling (CS) has been a topic of extensive research in various areas such as digital image processing [1], wireless channel estimation [2, 3], radar imaging [4], cognitive radio [5] etc. In CS, a significantly reduced number of measurements is obtained from the incoming data stream and is expected to be reconstructable from these small number of measurements [6]. This technique basically combines two key concepts: sparse representation with a choice of a linear basis for the class of desired signal and incoherent measurements of the considered signal to extract the maximum information using the minimum number of measurements [7]. Mathematical models required to implement $\mathrm{CS}$ include the development of new types of linear bases, $l_{1}$-optimisation to recover the sparse representations and the design of optimal dual measurements [8]. However, reconstructability of the signals exploiting CS is dependent on the following two principles: sparsity and incoherence nature of the signals [1]. The sparsity measure of natural continuous or discrete time signal provides the indication of information content sensitivity as well as the measure of degree of freedom of the signal. Incoherence indicates the duality between time and frequency, hence provides the concept that signals having sparse representations must be spread out in the domain in which they are acquired.

CS was originally used for efficient storage and compression of digital images $[1,6]$. CS theory states that certain signals can be recovered from far fewer samples or measurements than the samples required by traditional methods [9]. In sparse signals, most of the signal energy is concentrated in few non-zero coefficients. To apply CS theory, it is not necessary for the signal itself to be sparse, but can be compressible within sparse representations of the signal in known transform domain. According to the nature of signals as an example, smooth signals are sparse in the Fourier basis, whereas piecewise smooth signals are sparse in a wavelet basis [1]. The concept of quantisation in compressive measurements has been introduced in [10] and the reconstruction from CS measurements quantised to 1 bit per measurement has been considered. Different techniques for applying $\mathrm{CS}$ in various applications have been addressed in [11]. CS has been considered from a Bayesian perspective in [12] and it has been claimed that the results from Bayesian analysis are often sparser than previous CS solutions. In [13], CS technique has been applied to single target tracking and the feasibility of using compressively sensed and processed waveforms for reliable target tracking has been investigated. In [2], CS technique has been applied for sparse channel estimation and multi-carrier underwater acoustic communications, where the channel features are considered to be sparse. CS technique has also 
been applied for pilot-based channel estimation to reduce the pilot overhead burden by exploiting the coherent sparsity of the radio channels [14].

Design of appropriate transform matrices plays an important role in accurate recovery of the compressed signal. A new framework to construct fast and efficient sensing matrices for practical compressive sensing, called structurally random matrix, has been introduced in [15]. A greedy algorithm, called orthogonal matching pursuit (OMP), has been presented in [16]. A sorted random measurement matrix has been proposed in [17] to apply OMP in image signal recovery applications. Subspace pursuit algorithm for reconstruction of sparse signals with and without noisy perturbations has been proposed in [18]. In [19], significant performance gain has been demonstrated by exploiting more realistic signal models than the simple sparse and compressible models considered in much literature.

In digital communication systems, natural random signals generated by the users are non-sparse in nature. However, the most important aspect of being able to apply CS in digital communication is to investigate the practical feasibility of reconstruction of such natural non-sparse signal from compressive measurements. Then it would be feasible to perform other kinds of statistical signal processing at the receiver such as detection, estimation etc. Practically, in the majority of applications, data acquisition is based on Nyquist sampling theorem [20]. Since the signal bandwidth requirement is significantly high in case of video applications, the technology is not cost effective to achieve necessary processing rates in order to satisfy the Nyquist theorem. The existing practical solutions bandlimit the signals while preventing aliasing. Furthermore, there is a significant class of digital signals that are compressible, that is, it is not necessary to transmit all the data in order to get acceptable representation of the original information signal. Practical solutions introduce lossy compressive processing at the source level [21].

In the existing literature, CS techniques have been used for different applications which mainly deal with sparse signals [7-11]. In this paper, a framework is proposed to apply CS technique for natural non-sparse random signals in the context of practical applications to digital communication. To evaluate the performance of the proposed framework, a multiple input multiple output orthogonal frequency division multiplexing (MIMO-OFDM) system with CS is considered. Within the assumed model, MIMO system exploits the spatial multiplexing technique by creating independently faded paths between transmit and receive antennas as in [22]. OFDM is a multi-carrier modulation technique, which provides higher spectral efficiency in comparison with the traditional modulation schemes [23]. MIMO-OFDM has been considered as an important technology in upcoming high data rate systems such as $4 \mathrm{G}$, IEEE 802.16, IEEE 802.11n, WiMax Mobile, WiMax Fixed and 3GPP LTE [24]. In the context of a MIMO-OFDM system, a CS-based channel estimation method for MIMO-OFDM systems over frequency-selective fading channel has been presented in [3], and it has been claimed that the proposed method outperforms the conventional least square method and greatly decreases the pilot overhead burden. In this paper, we apply the CS technique with the objective of enhancing spectrum efficiency of the wireless communication systems.

In [25], code division multiplexing (CDM) technique has been applied in the transmitter side and reverse process has been performed in the receiver side of the MIMO-OFDM system. In contrast to the principle applied in [25], we apply reverse process, that is, compression in the transmitter side and decompression in the receiver side in this work. A measurement matrix based on Hadamard matrix is proposed to carry out CS for natural random signals. The main contribution of this research work is the exploration of a new random measurement matrix to perform CS with lower degradation in QoS, so that the possibility of the occurrence of ambiguous conditions at the receiver is eliminated. According to authors' knowledge, no contributions have been reported in the literature using this concept. Finally, the performance evaluation of the proposed framework is carried out in terms of bit error rate (BER) against bit energy-to-noise ratio $\left(E_{\mathrm{b}} / N_{\mathrm{o}}\right)$ and the normalised spectrum efficiency against $E_{\mathrm{b}} / N_{\mathrm{o}}$.

The rest of this paper is organised as follows: Section 2 provides general overview of the CS technique. Section 3 provides theoretical basis on compressibility of natural random signals and then defines the proposed framework to implement CS on to a natural random signal. Section 4 provides the analysis of the conditional probability on accurate detectability of the signals. Section 5 presents a system-level evaluation of the proposed framework to obtain a measure of compressibility of natural digital signal for an acceptable QoS considering a simple MIMO-OFDM system. Simulation model and numerical results are presented in Section 6. Section 7 concludes this paper.

\subsection{Notation}

Throughout the formulations of this paper, boldface upper and lower case letters are used to denote matrices and vectors, respectively, $\mathbb{E}[\cdot]$ denotes expectation, $\mathcal{R}$ denotes the real plane, $(\cdot)^{\mathrm{T}}$ denotes the transpose, $(\cdot)^{\mathrm{H}}$ denotes the conjugate transpose and $\boldsymbol{I}$ denotes the identity matrix.

\section{Overview of CS}

Sampling is one of the fundamental steps carried out at modern digital communication receiver. According to Nyquist sampling theorem, no information loss is expected with the reconstructed signal if sampling rate is twice the bandwidth or maximum frequency component of the signal depending on whether the signal is bandpass or bandlimited [20]. There are different sampling trends emerging in this field such as faster sampling, larger dynamic range, higher-dimensional data, lower energy consumption and new sampling modalities such as CS [7]. The CS technique performs sampling at a rate less than the Nyquist rate and reconstructs the original signal back from significantly lower number of compressive measurements. The CS theory states that certain signals can be recovered from far fewer samples or measurements than the samples required by traditional methods [9]. To apply CS theory, it is not necessary for the signal itself to be sparse and it can be applied by transforming a non-sparse signal into a sparse form in some known transform domain. If an $N$-dimensional signal can be converted to a sparse form with only $K$ non-zero coefficients and $K \ll N$, the signal can be regarded as a $K$-sparse signal. In other words, if the largest $K$ coefficients in the sparse form can retrieve an acceptable approximation of the signal and $K \ll N$, the signal can be considered as a compressible signal. Such compression schemes can reduce the dimensionality of an 
$N$-dimensional sparsely represented signal $\boldsymbol{x}$ from the natural set of coefficients $\boldsymbol{\alpha}$ in a basis expansion $\boldsymbol{x}=\boldsymbol{\Psi} \boldsymbol{\alpha}$, with $\boldsymbol{\Psi}$ an $N \times N$ basis matrix [19], where every set of $N$ coefficients $\left\{\alpha_{i}\right\}_{i=1}^{N}$ can be represented in terms of a signal vector $\boldsymbol{x} \in \mathcal{R}^{N}$ within a given basis matrix $\boldsymbol{\Psi}$.

Suppose $\overline{\boldsymbol{x}}$ be a measurement vector of size $M$ and it is represented with fewer samples in terms of signal vector $\boldsymbol{x}$ of size $N(M<N)$ through some random projection, where $M$ is constrained by $M \leq K \log N$ [21], and $K$ is the number of non-zero coefficients, the measurement vector $\overline{\boldsymbol{x}}$ can be written as

$$
\overline{\boldsymbol{x}}=\boldsymbol{\Phi} \boldsymbol{x}=\boldsymbol{\Phi} \Psi \alpha
$$

where $\boldsymbol{\Phi}$ is a $M \times N$ random measurement matrix and it must obey uniform uncertainty principle to guarantee the reconstruction of the signal [26]. The measurement matrix is unstructured and universally incoherent to the basis matrix $\boldsymbol{\Psi}$ and with every measurement, fractional information about the sparse coefficients can be obtained. Hence, it does not have to match any structure of the signal but looks more like random noise than any feature of the signal vector [1]. Standard CS theory dictates that robust signal recovery is possible from $M=O \quad[K \log (N / K)]$ measurements [19]. Many contributions related to CS till date have been carried out for sparse signals [6, 21, 27]. In this paper, the application of CS for natural non-sparse random information signal for digital wireless communication is considered.

\section{Proposed compression algorithm}

\subsection{Compressibility of natural random signals}

A signal, which can be represented with a fewer number of samples, less than the length of the signal can be considered as a compressed signal. These types of signals are pervasive in real applications. Natural signals such as audio and speech signals are highly compressible. Image, music, speech compression algorithms and coders are important parts of software in many technologies, from desktop computers to MP3 players. Many types of automatically generated signals are also highly redundant [28]. As defined in the earlier section, a set of $K$-sparse signal implies that only $K(\ll N)$ of the coefficients $\alpha$ are non-zero. On the other hand, compressibility implies that coefficients $\alpha$, when sorted, decay rapidly enough to zero so that $\alpha$ can be represented with $K$ number of samples which are reconstructable in $N$ dimensions.

Recent results have shown that a relatively small number of random projections of a signal can contain most of its salient information [19]. Many natural and man-made signals are not purely sparse, but can be approximated as compressible. Natural signals or images are sparse (or compressible) in the discrete cosine transform (DCT) domain, that is, many DCT coefficients are zero or small enough to be approximated by zero.

Let us consider a natural signal vector $\boldsymbol{x}$ whose coefficients, when sorted in order of decreasing magnitude in transform domain $S$, decay according to the following power law [19]

$$
\left|\boldsymbol{x}_{\mathcal{I}(q)}\right| \leq S q^{-1 / r}, \quad q=1,2, \ldots, N
$$

where $\mathcal{I}$ indexes the sorted coefficients and $r$ is the order of the decay. Owing to the rapid decay of their coefficients, such signals are well approximated as $K$-sparse signals [19] by considering only $K$ number of the highest coefficients to be non-zero in the signal vector. Let $\boldsymbol{x}_{K} \in \Sigma_{K}$ represent the best $K$-term approximation of $\boldsymbol{x}$, which is obtained by keeping only the first $K$-terms in $x_{\mathcal{I}(q)}$ in (2). The error of this approximation can be denoted as $\sigma_{K}$, which in the $l_{p}$-norm can be written as

$$
\sigma_{K}(\boldsymbol{x})_{p}=\left\|\boldsymbol{x}-\boldsymbol{x}_{K}\right\|_{p}
$$

where the $l_{p}$-norm of the vector $\boldsymbol{x}$ is defined as $\|\boldsymbol{x}\|_{p}=\left(\sum_{q=1}^{N}\left|\boldsymbol{x}_{q}\right|^{p}\right)^{1 / p}$ for $0<p<\infty, p$ being order of normalisation. Then the above equation can be rewritten as [19]

$$
\sigma_{K}(\boldsymbol{x})_{p} \leq(r s)^{-1 / p} S K^{-s}, \quad \text { for } \quad r<p
$$

with $s=(1 / r)-(1 / p)$. In terms of $l_{p}$-norm, the signal's best approximation error has a power-law decay with exponent $s$ as $K$ increases. The natural random signal which satisfies (4) can be said to be $s$-compressible [19].

The approximation of compressibility of the natural signals for digital communication solely depends on the selection of transform domain basis for sparse representation of the signal. In this proposed framework, full $N$-sample signal $\boldsymbol{x}$ can be acquired. The product of the orthogonal basis along with the measurement matrix is assumed to be a subset of Hadamard matrix, that is, $\boldsymbol{H}_{\mathrm{c}}=\boldsymbol{\Phi} \boldsymbol{\Psi}$ in relation to (1). Therefore natural random signals can be compressed by representing them in the proposed transform domain.

\subsection{Proposed compressive transmission framework (CTF)}

Many contributions related to $\mathrm{CS}$ have been carried out for sparse signals $[1,6,21]$. As stated earlier, the compression of a non-sparse signal depends on the proper selection of measurement matrix. In this work, a subset of Hadamard matrix has been used as an orthogonal compressing measurement matrix. The chip rate to generate an $\mathrm{N}$-dimensional Hadamard matrix, denoted as $\boldsymbol{H}$, is assumed to be the same as bit rate.

From this point, we consider $\boldsymbol{x}$ to be a binary information sequence vector of size $(N \times 1)$ arriving at the transmitter and $\boldsymbol{x}_{\mathrm{c}}$ be the compressed signal vector of dimension $(M \times$ 1) obtained after multiplying the incoming sequence with the compressive measurement matrix $\boldsymbol{H}_{\mathrm{c}}$ of dimension $(M \times$ $N$ ), which is a subset of $\boldsymbol{H}$, that is, $\boldsymbol{H}_{\mathrm{c}} \in \boldsymbol{H}$, where $M<N$. The compressed information sequence $\boldsymbol{x}_{\mathrm{c}}$ that is to be transmitted can be written as

$$
\boldsymbol{x}_{\mathrm{c}}=\boldsymbol{H}_{\mathrm{c}} \boldsymbol{x}
$$

This compressed signal can be passed through channel using different transmission techniques. The noisy received signal after passing through Gaussian channel can be written as

$$
\boldsymbol{y}=\boldsymbol{x}_{\mathrm{c}}+\boldsymbol{w}
$$

where $\boldsymbol{w}$ is Gaussian noise vector. At the receiver, the information signal can be recovered by passing through a zero forcing $(\mathrm{ZF})$ or minimum mean square error (MMSE) 
detectors.

$$
\hat{\boldsymbol{x}}=\frac{1}{N} \boldsymbol{G} \boldsymbol{y}
$$

where $\boldsymbol{G}=\left(\boldsymbol{H}_{\mathrm{c}}^{\mathrm{H}} \boldsymbol{H}_{\mathrm{c}}\right)^{-1} \boldsymbol{H}_{\mathrm{c}}^{\mathrm{H}}$ and $\boldsymbol{G}=\left(\boldsymbol{H}_{\mathrm{c}}^{\mathrm{H}} \boldsymbol{H}_{\mathrm{c}}+\sigma_{w}^{2} \boldsymbol{I}\right)^{-1} \boldsymbol{H}_{\mathrm{c}}^{\mathrm{H}}$ for $\mathrm{ZF}$ and MMSE detectors.

In case of non-sparse signals, the above estimation results are ambiguous for the case of the input sequence to be all 1 's and all 0's. Hence, a new operator has been introduced to avoid such ambiguity. This proposed operator is to be generated from a lower triangular matrix with its all non-zero components as 1 's denoted as $\boldsymbol{L}$, of dimension $(N \times N)$. The inverse of this lower triangular matrix yields the difference matrix $(\boldsymbol{D})$, that is, $\boldsymbol{D}=\boldsymbol{L}^{-1}$. The modified compressive measurement matrix is denoted by $\boldsymbol{H}_{\mathrm{d}}$ and can be defined as

$$
\boldsymbol{H}_{\mathrm{d}}=\boldsymbol{D} \boldsymbol{H}_{\mathrm{c}}
$$

Then, the compressive signal with modified measurement matrix can be written as: $\boldsymbol{x}_{\mathrm{c}}^{\mathrm{d}}=\boldsymbol{H}_{\mathrm{d}} \boldsymbol{x}$. Hence the noisy received signal is expressed as

$$
\boldsymbol{y}=\boldsymbol{x}_{\mathrm{c}}^{\mathrm{d}}+\boldsymbol{w}=\boldsymbol{H}_{\mathrm{d}} \boldsymbol{x}+\boldsymbol{w}
$$

At the receiver, information signal can be recovered using the following relation.

$$
\overline{\boldsymbol{x}}=\frac{1}{N} \overline{\boldsymbol{G}} \boldsymbol{y}
$$

where $\overline{\boldsymbol{G}}=\left(\boldsymbol{H}_{\mathrm{d}}^{\mathrm{H}} \boldsymbol{H}_{\mathrm{d}}\right)^{-1} \boldsymbol{H}_{\mathrm{d}}^{\mathrm{H}}$ and $\overline{\boldsymbol{G}}=\left(\boldsymbol{H}_{\mathrm{d}}^{\mathrm{H}} \boldsymbol{H}_{\mathrm{d}}+\sigma_{w}^{2} \boldsymbol{I}\right)^{-1} \boldsymbol{H}_{\mathrm{d}}^{\mathrm{H}}$ for ZF and MMSE detectors.

\section{Symbol detection reliability test}

For the sake of simplicity, the detection reliability of the signal at the receiver is analysed with generalised likelihood ratio test (GLRT) with first-order statistics [29]. The Gaussian probability density function for a random variable $x$ can be defined as

$$
\begin{aligned}
p(x)= & \frac{1}{\sqrt{2 \pi \sigma^{2}}} \exp \left[-\frac{1}{2 \sigma^{2}}(x-\mu)^{2}\right], \\
& -\infty<x<\infty
\end{aligned}
$$

where $\mu$ is the mean and $\sigma^{2}$ is the variance of $x$. It is denoted by $\mathcal{N}\left(\mu, \sigma^{2}\right)$. Let $\mathbb{H}_{0}$ and $\mathbb{H}_{1}$ denote the hypotheses of noise-only case and signal plus noise case, respectively. For the detection of a signal in additive white Gaussian noise channel, Neyman-Pearson (NP) criterion can be used to design an optimum detection problem of distinguishing between two hypotheses in the following way

$$
\begin{aligned}
\mathfrak{H}_{0}: y[k] & =w[k], & k & =0,1, \ldots, N-1 \\
\mathfrak{H}_{1}: y[k] & =x[k]+w[k], & k & =0,1, \ldots, N-1
\end{aligned}
$$

where the signal amplitude of $x[k]$ is assumed to be known, $y[k]$ is the $k$ th observation of received noisy signal, $w[k]$ is the white noise with variance $\sigma^{2} . w[k]$ can be taken as a zero-mean Gaussian noise process with autocorrelation function

$$
r_{w w}[k]=\mathbb{E}(w[k] w[k+n])=\sigma^{2} \delta[n]
$$

where $\delta[n]$ is the discrete delta function. The NP detector for a deterministic signal vector $\boldsymbol{y}$ decides $\mathbb{W}_{1}$ if the likelihood ratio exceeds a dynamically controlled threshold $\gamma$. That is

$$
L[x]=\frac{p\left(y ; \mathbb{H}_{1}\right)}{p\left(y ; \mathbb{H}_{0}\right)}>\gamma
$$

where $\boldsymbol{y}=[y(0), y(1), \ldots, y(N-1)]^{\mathrm{T}}$. The conditional probabilities $p\left(\boldsymbol{y} ; \mathbb{H}_{1}\right)$ and $p\left(\boldsymbol{y} ; \mathbb{H}_{0}\right)$ can be written as

$$
\begin{gathered}
p\left(y ; \mathbb{H}_{1}\right)=\frac{1}{\left(2 \pi \sigma^{2}\right)^{N / 2}} \exp \left[-\frac{1}{2 \sigma^{2}} \sum_{k=0}^{N-1}(y[k]-x[k])^{2}\right] \\
p\left(y ; \mathbb{H}_{0}\right)=\frac{1}{\left(2 \pi \sigma^{2}\right)^{N / 2}} \exp \left[-\frac{1}{2 \sigma^{2}} \sum_{k=0}^{N-1} y^{2}[k]\right]
\end{gathered}
$$

Combining (14), (15) and (16), we can obtain the following equation

$$
L(\boldsymbol{y})=\exp \left[-\frac{1}{2 \sigma^{2}}\left(\sum_{k=0}^{N-1}(y[k]-x[k])^{2}-\sum_{n=0}^{N-1} y^{2}[k]\right)\right]>\gamma
$$

In ideal decision rule, it can be considered that all the parameters are known and the problem reduces to decide between one of the following two complex Gaussian hypotheses [30]

$$
\begin{aligned}
& \mathbb{H}_{0}: \boldsymbol{y} \sim \mathcal{N}\left(0, \operatorname{diag}\left(\sigma_{1}^{2}, \ldots, \sigma_{K}^{2}\right)\right) \\
& \mathbb{H}_{1}: \boldsymbol{y} \sim \mathcal{N}\left(0, \operatorname{diag}\left(\tau_{1}^{2}, \ldots, \tau_{K}^{2}\right)\right)
\end{aligned}
$$

Applying above threshold equation to this scenario, the following ideal decision equation can be obtained

$$
L(y)=\sum_{k=1}^{K}\left[\frac{1}{2}\left(\frac{\sigma_{k}^{2}}{\tau_{k}^{2}}-1\right) \frac{2\left|y_{k}\right|^{2}}{\sigma_{k}^{2}}-\ln \frac{\sigma_{k}^{2}}{\tau_{k}^{2}}\right]
$$

where $y_{k}$ is the $k$ th component of observation vector $y$. If the true parameters $\sigma_{k}^{2}$ and $\tau_{k}^{2}$ are known, the GLRT can be used. In this scenario, the maximum likelihood estimates of these parameters are substituted into optimal rule in place of unknown values. Let $S_{k_{1}}^{2}=\sum_{l=1}^{N_{s}}\left|v_{k, l}\right|^{2}$ and $S_{k_{0}}^{2}=\sum_{l=1}^{N_{T}}\left|w_{k, l}\right|^{2}$, where $v_{k, l}$ and $w_{k, l}$ represent the $k$ th components of observations drawn under $\mathbb{H}_{1}$ and $\mathbb{H}_{0}$, respectively, and $N_{s}$ and $N_{T}$ denotes the number of samples under respective hypothesis. Then, $2 N_{s} S_{k_{1}}^{2} / \sigma_{k}^{2}$ and $2 N_{T} S_{k_{0}}^{2} / \sigma_{k}^{2}$ follow $\chi^{2}$ distributions with $2 N_{s}$ and $2 N_{T}$ degrees of freedom, respectively. Let us denote the $k$ th observation of a received symbol with $S_{k_{0}}$ and $S_{k_{1}}$ for null and alternate hypothesis, respectively. The average noise power is denoted by $\sigma_{w}^{2}$, the variances of the transmitted sequence without and with the CTF are denoted as $\sigma_{x}^{2}$ and $\sigma_{x_{\mathrm{c}}}^{2}$, respectively. Assuming unity degree of freedom, the plug in decision parameter for the $k$ th observation of transmitted signal without compression, 
denoted by $L_{k}$, can be written as

$$
L_{k}=\left[\left(\frac{\sigma_{w}^{2}}{S_{k_{1}}}-\frac{\sigma_{w}^{2}}{S_{k_{0}}}\right) \frac{\sigma_{x}^{2}}{\sigma_{w}^{2}}-\ln \left(\frac{S_{k_{0}}}{S_{k_{1}}}\right)\right] \gtrless_{\mathbb{H}_{0}}^{\mathbb{H}_{1}} \gamma
$$

The decision parameter on the detectability of the signal, while using the proposed CTF with compression ratio $M / N$, denoted by $L_{k}^{\mathrm{c}}$, can be written as

$$
L_{k}^{\mathrm{c}}=\left[\frac{M}{N}\left(\frac{\sigma_{w}^{2}}{S_{k_{1}}}-\frac{\sigma_{w}^{2}}{S_{k_{0}}}\right) \frac{\sigma_{x_{\mathrm{c}}}^{2}}{\sigma_{w \mathrm{c}}^{2}}-\ln \left(\frac{S_{k_{0}}}{S_{k_{1}}}\right)\right] \gtrless_{\mathbb{H}_{0}}^{\mathbb{H}_{1}} \gamma
$$

Let $p\left(\mathbb{H}_{i}, \mathbb{H}_{j}\right)$ denote the probability of deciding $\mathbb{H}_{i}$ when $\mathbb{H}_{j}$ is true with $\{i, j\} \in\{0,1\}$. Then the term $p\left(\mathbb{H}_{1}, \mathbb{H}_{0}\right)$ indicates the probability of deciding $\mathbb{H}_{1}$ when $\mathbb{H}_{0}$ is true, referred to as probability of false detection $\left(p_{\mathrm{fa}}\right)$. For optimum detection, $p\left(\mathbb{H}_{0}, \mathbb{H}_{1}\right)$ needs to be minimised, that is, $1-p\left(\mathbb{H}_{0}, \mathbb{W}_{1}\right)$ termed as probability of detection $\left(p_{\mathrm{d}}\right)$, needs to be maximised. Therefore $p_{\mathrm{d}}$ can be written as

$$
p_{\mathrm{d}}=p\left(\mathbb{H}_{1} ; \mathbb{H}_{1}\right)=\operatorname{Pr}\left\{L_{k}^{\mathrm{c}}>\gamma ; \mathbb{H}_{1}\right\}
$$

where $\operatorname{Pr}\{\cdot\}$ indicates the probability.

\section{System-level evaluation of proposed framework}

A MIMO-OFDM system with $N_{\mathrm{T}}$ transmit and $N_{\mathrm{R}}$ receive antennas is considered. The block diagrams of simple MIMO-OFDM transmitter and receiver in the context of proposed compressive framework are shown in Figs. 1 and 2, respectively. Similar communication model has been considered in [25]. A data stream of length $J$ is divided in to $P$ number of sub-blocks, each of length $N$ symbols and each data stream is denoted by matrix $\boldsymbol{S}=\left[\begin{array}{llll}\boldsymbol{s}_{1} & \boldsymbol{s}_{2} & \cdots & \boldsymbol{s}_{P}\end{array}\right]$ of dimension $(N \times P)$, where the information symbol sub-block $s_{i}=\left[\begin{array}{lll}s_{i}(1) & s_{i}(2) & \cdots\end{array}\right.$ $\left.s_{i}(N)\right]^{\mathrm{T}}$ with $i \in\{1,2, \ldots, P\}$. For compression purpose, each data sub-block is multiplied with compressive measurement matrix $\boldsymbol{H}_{\mathrm{d}}$ of dimension $(Q \times N)$ as defined in (8), where $Q<N$. This results in a compressed information symbol matrix $\boldsymbol{X}=\left[\begin{array}{llll}\boldsymbol{x}_{1} & \boldsymbol{x}_{2} & \cdots & \boldsymbol{x}_{P}\end{array}\right] \quad$ with $\boldsymbol{x}_{i}=\left[\begin{array}{llll}x_{i}(1) & x_{i}(2) & \cdots & x_{i}(Q)\end{array}\right]^{\mathrm{T}}$. Hence

$$
\boldsymbol{x}_{i}=\boldsymbol{H}_{\mathrm{d}} \boldsymbol{s}_{i}
$$

Subsequently, the total data stream of $J(N \times P)$ is compressed into $J_{1}(Q \times P)$ number of symbols; then mapped into $J_{1} / N_{c}$ blocks, each of the length $N_{c}$. It is assumed to have $N_{c}$ number of sub-carriers within each OFDM symbol. The resulting compressed symbols are interleaved before serial to parallel converter, followed by conversion to OFDM symbol for each of $N_{\mathrm{T}}$ transmit antennas. Furthermore, within each OFDM symbol, a cyclic prefix of length $C_{p}$ is inserted to avoid intersymbol interference and intercarrier interference. The length of the cyclic prefix is assumed to be longer than maximum delay spread of the radio channel. The MIMO channel matrix considered in this work can be expressed as: $\overline{\boldsymbol{H}}=\left[\begin{array}{llll}\overline{\boldsymbol{h}}_{1} & \overline{\boldsymbol{h}}_{2} & \cdots & \overline{\boldsymbol{h}}_{N_{\mathrm{R}}}\end{array}\right]^{\mathrm{T}}$, where each vector $\overline{\boldsymbol{h}}_{j}$ can be written as: $\overline{\boldsymbol{h}}_{j}=\left[\begin{array}{llll}h_{j 1} & h_{j 2} & \cdots & h_{j N_{\mathrm{T}}}\end{array}\right]$, where $h_{j, k}$ represents the channel fading coefficient between $k$ th transmit antenna and $j$ th receive antenna. The received symbol vector at $l$ th block of received symbols can be denoted by $\boldsymbol{r}_{l}=\left[\begin{array}{llll}r_{l}(1) & r_{l}(2) & \cdots & r_{l}\left(N_{\mathrm{R}}\right)\end{array}\right]^{\mathrm{T}}$, where $l \in\{1$, $2, \ldots, P\}$ and a received symbol corresponding to a symbol of $l$ th transmitted block can be written as

$$
r_{l}(n)=\sum_{j=1}^{N_{\mathrm{R}}} h_{j l} x_{l}(n)+w_{l}(n)
$$

where $x_{l}(n)$ is $n$th symbol of $l$ th block of transmitted symbols and $\boldsymbol{w}_{l}=\left[\begin{array}{llll}w_{l}(1) & w_{l}(2) & \cdots & w_{l}\left(N_{\mathrm{R}}\right)\end{array}\right]^{\mathrm{T}}$ is the Gaussian noise vector for $l$ th block of received symbols. The independent and identically distributed Gaussian noise is with zero mean and variance $\sigma_{w}^{2}$, so that $\mathbb{E}\left[\boldsymbol{w}_{l} \boldsymbol{w}_{l}^{\mathrm{H}}\right]=\sigma_{w}^{2} \boldsymbol{I}$. Besides that the input data-stream is uncorrelated with the property $\mathbb{E}\left[s_{i} s_{i}^{\mathrm{H}}\right]=\boldsymbol{I}$. Hence the overall received symbol matrix becomes $\boldsymbol{R}=\left[\begin{array}{llll}\boldsymbol{r}_{1}^{\mathrm{T}} & \boldsymbol{r}_{2}^{\mathrm{T}} & \cdots & \boldsymbol{r}_{P}^{\mathrm{T}}\end{array}\right]$ in response to the initial targeted data block $\boldsymbol{S}$. Therefore the received symbol matrix $\boldsymbol{R}$ can also be written as

$$
\begin{aligned}
\boldsymbol{R} & =\overline{\boldsymbol{H}} \boldsymbol{X}+\boldsymbol{W} \\
& =\overline{\boldsymbol{H}} \boldsymbol{H}_{\mathrm{d}} \boldsymbol{S}+\boldsymbol{W} \\
& =\boldsymbol{C S}+\boldsymbol{W}
\end{aligned}
$$

where $\boldsymbol{C}=\overline{\boldsymbol{H}}_{\mathrm{d}}$. In a MIMO receiver, each antenna receives signal from all transmitting antennas and different combining techniques can be used to combine signals received by different antennas. At each receiving antenna branch, the received signal is passed through radio frequency down converter to bring down the high frequency into lower one. Then the signal is passed through digital-to-analogue converter to generate a digital bit stream of the received signal. For simplicity, a ZF equaliser has been used to detect and reconstruct the original transmitted signal. The reconstructed signal matrix $\hat{\boldsymbol{S}}$ can be written as

$$
\hat{\boldsymbol{S}}=\frac{1}{N} \overline{\boldsymbol{G}} \boldsymbol{R}
$$

where $\boldsymbol{R}$ is the received signal matrix and $\overline{\boldsymbol{G}}$ is ZF equaliser coefficient given by; $\overline{\boldsymbol{G}}=\left(\boldsymbol{C}^{\mathrm{H}} \boldsymbol{C}\right)^{-1} \boldsymbol{C}^{\mathrm{H}}$ with $\boldsymbol{C}=\overline{\boldsymbol{H}} \boldsymbol{H}_{\mathrm{d}}$.

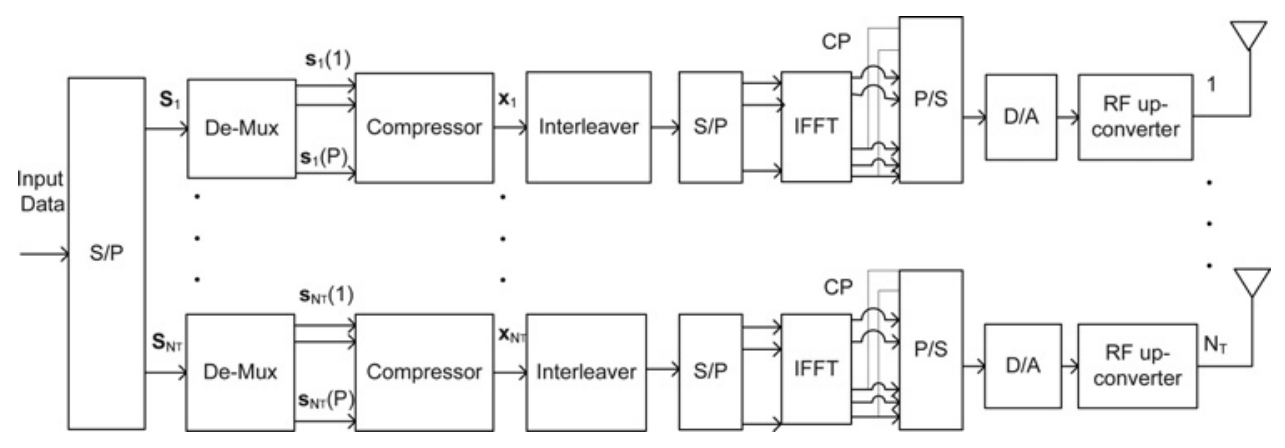

Fig. 1 System model for the proposed transmitter 


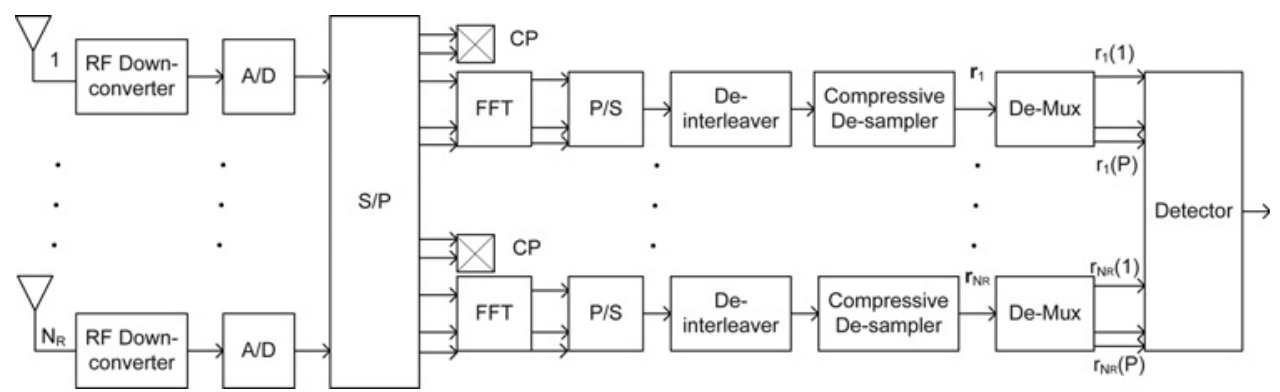

Fig. 2 System model for the proposed receiver

\section{Simulation and results}

The performance of the proposed framework in the context of a simple MIMO-OFDM system has been evaluated in terms of BER against $\left(E_{\mathrm{b}} / N_{\mathrm{o}}\right)$. The proposed system has been simulated with a forward error correction (FEC) code and without a FEC code. A convolution code with $1 / 2$ rate has been used for FEC purpose. The dimensions of $\boldsymbol{H}$ and $\boldsymbol{H}_{\mathrm{d}}$ are assumed to be $(4 \times 4)$ and $(4 \times 3)$, respectively, to obtain $25 \%$ compression. The chip rate of Hadamard matrix is considered to be equal to the bit rate considered. Furthermore, compression with compression ratio of 3/4 has been applied to the incoming data stream. Randomly generated frames with 30 bits/frame are encoded using a convolutional encoder.

The proposed compressive transmission scheme has been compared with traditional amplitude shift keying (ASK) and frequency shift keying (FSK) schemes. Phase shift keying (PSK) modulation scheme has been considered for the evaluation of proposed compressive framework because of its simplicity. Fig. 3 shows the comparative result of BER performance of the proposed CTF with traditional ASK and FSK transmission schemes. From the same figure it is observed that the proposed compressive transmission scheme without FEC requires only $1.5 \mathrm{~dB}$ more $E_{\mathrm{b}} / N_{\mathrm{o}}$ in comparison with the traditional ASK and PSK at BER value of $10^{-3}$.

Fig. 4 shows comparative results of the proposed CTF with FEC and the CTF without FEC. From the simulation results, it can be observed that almost $4 \mathrm{~dB}$ more $E_{\mathrm{b}} / N_{\mathrm{o}}$ is needed in case of the proposed framework as compared with the

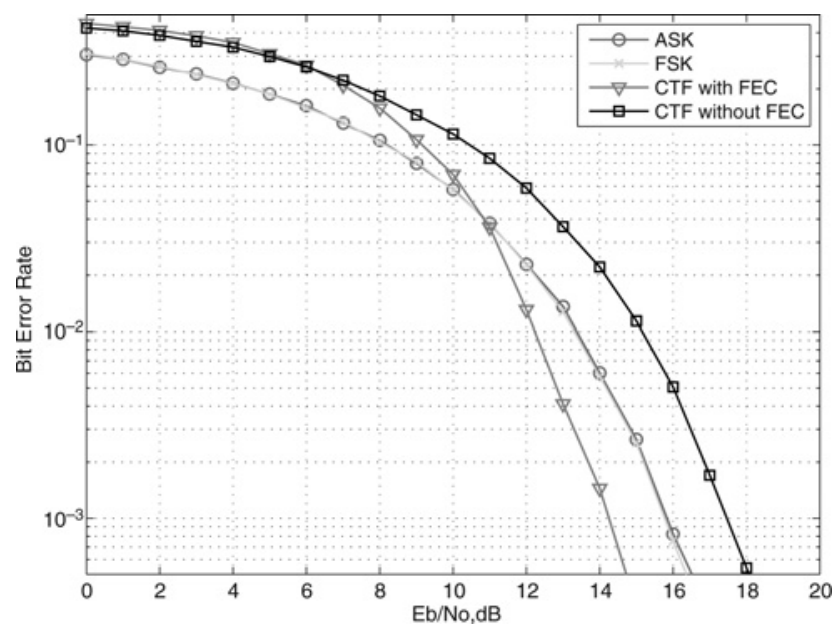

Fig. 3 Comparison of compressive technique with traditional transmission schemes traditional conventional PSK transmission scheme at BER value of $10^{-3}$. This difference remains consistent in both cases with and without FEC beyond the required value of $10^{-2}$. From the simulation results, it can be claimed that the proposed CTF scheme can achieve $25 \%$ saving in spectrum by sacrificing almost $4 \mathrm{~dB} E_{\mathrm{b}} / N_{\mathrm{o}}$. By varying the nature of measurement matrix $\boldsymbol{H}_{\mathrm{d}}$ chosen in this work, this QoS trade-off can be optimised since the gain in spectrum efficiency and requirement of more energy depends on the selection of matrix $\boldsymbol{H}_{\mathrm{d}}$.

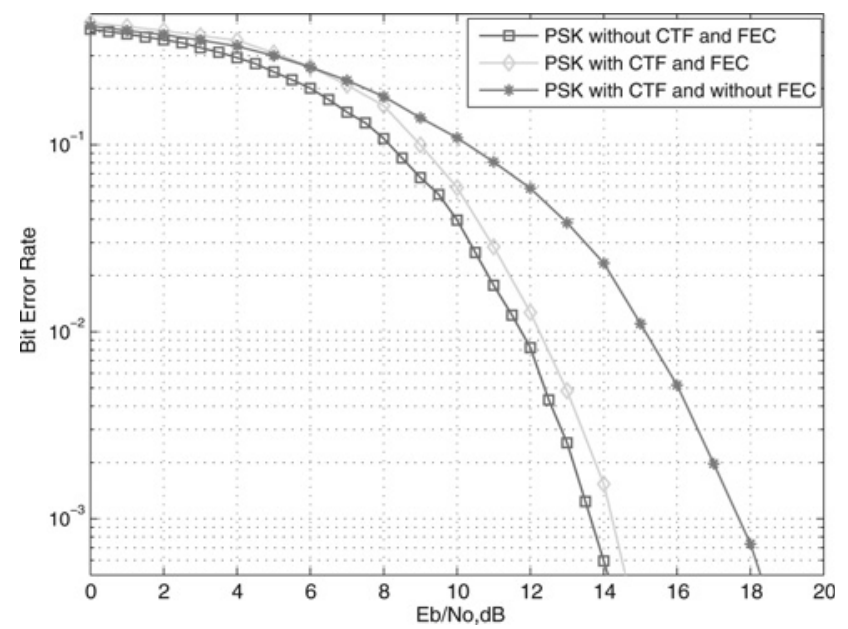

Fig. 4 Comparison of proposed framework with and without FEC

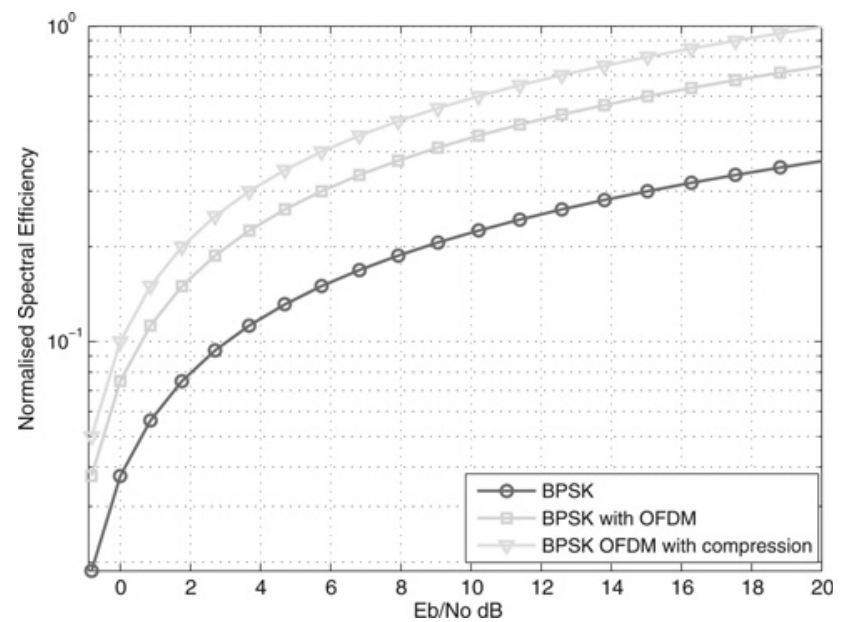

Fig. 5 Comparison of normalised spectral efficiency for different transmission schemes 
Furthermore, the normalised spectral efficiency of CS technique has been compared with spectral efficiency of OFDM transmission technique and basic binary phase shift keying transmission scheme. The comparison result has been shown in Fig. 5. From the simulation result, it can be noted that the proposed compressive scheme can provide $25 \%$ enhanced spectral efficiency for a bandwidth constraint application, trading off with $4 \mathrm{~dB} E_{\mathrm{b}} / N_{\mathrm{o}}$ for $10^{-3}$ BER.

\section{Conclusion}

A CTF exploiting the basis of CS has been proposed in the context of practical implementation feasibility within wireless communication systems. The proposed framework has been evaluated in the context of a simple MIMO-OFDM system. The simulation results show that the proposed framework can achieve $25 \%$ saving in spectrum with the cost of $4 \mathrm{~dB} E_{\mathrm{b}} / N_{\mathrm{o}}$ at BER value of $10^{-3}$. The proposed framework can also save $25 \%$ hardware resources if the data rate required is to be the same. Furthermore, it has been noted that this result is consistent with channel coding and without channel coding. It can be concluded that the proposed transmission scheme can achieve spectrum as well as hardware resources (e.g. number of transmit/receiver antennas) saving by sacrificing some amount of transmitted power. Therefore this technique can be used effectively in the spectrum limited applications. Designing an adaptive measurement matrix remains an open challenge for the proposed framework. Analysing the compressibility of non-sparse signals and the extension of this model using different channel coding, efficient channel estimation and detection techniques can be the future work in this area.

\section{References}

1 Candes, E.J., Wakin, M.: 'An introduction to compressive sampling', IEEE Signal Process. Mag., 2008, 25, (2), pp. 21-30

2 Berger, C.R., Wang, Z., Huang, J., Zhou, S.: 'Application of compressive sensing to sparse channel estimation', IEEE Commun. Mag., 2010, 48, (11), pp. 164-174

3 Peng, Y., Yang, X., Zhang, X., Wang, W., Wu, B.: 'Compressed MIMO-OFDM channel estimation'. Proc. 12th IEEE Int. Conf. Communication Technology, November 2010, pp. 1291-1294

4 Baraniuk, R., Steeghs, P.: 'Compressive radar imaging'. Proc. IEEE Radar Conf., April 2007, pp. 128-133

5 Tian, Z., Giannakis, G.B.: 'Compressed sensing for wideband cognitive radios'. IEEE Int. Conf. Acoustics, Speech and Signal Processing, April 2007, vol. 4, pp. 1357-1360

6 Candes, E.J.: 'Compressive sampling'. Proc. Int. Congress of Mathematicians, 2006, vol. 3, pp. 1433-1452

7 Romberg, J., Wakin, M.: 'Compressed sensing: a tutorial'. IEEE Statistical Signal Processing Workshop, Wisconsin, 2007
8 Lamoureux, M.: 'Tutorial on compressive sampling'. University of Calgary, Calgary, available online, website: http://www.cspg.org/ conventions/

9 Ciancio, A., Pattem, S., Ortega, A., Krishnamachari, B.: 'Energy-efficient data representation and routing for wireless sensor networks based on a distributed wavelet compression algorithm'. Proc. Int. Conf. Information Processing in Sensor Networks, 2006, pp. 309-316

10 Boufounos, P.T., Baraniuk, R.G.: '1-Bit compressive sensing'. Proc. 42nd Annual Conf. Information Sciences and Systems, March 2008

11 Mishali, M., Eldar, Y.C.: 'From theory to practice: sub-Nyquist sampling of sparse wideband analog signals', IEEE J. Sel. Top. Signal Process., 2010, 4, (2), pp. 2941-2946

12 Shihao, J., Ya, X., Carin, L.: 'Bayesian compressive sensing', IEEE Trans. Signal Process., 2008, 56, (6), pp. 2346-2356

13 Kyriakides, K.: 'Radar tracking performance when sensing and processing compressive measurements'. 13th Conf. Information Fusion, July 2010

14 Li, W., Preisig, J.C.: 'Estimation of rapidly time-varying sparse channels', IEEE J. Ocean. Eng., 2007, 32, (10), pp. 927-939

15 Do, T.T., Gan, L., Nguyen, N.H., Tran, T.D.: 'Fast and efficient compressive sensing using structurally random matrices', IEEE Trans. Signal Process., 2012, 60, (1), pp. 139-154

16 Tropp, J., Gilbert, A.: 'Signal recovery from random measurements via orthogonal matching pursuit', IEEE Trans. Inf. Theory, 2007, 53, (12), pp. $4655-4666$

17 Wang, Z., Lee, I.: 'Sorted random matrix for orthogonal matching pursuit'. Proc. Int. Conf. Digital Image Computing: Techniques and Applications, December 2010, pp. 116-120

18 Dai, W., Milenkovic, O.: 'Subspace pursuit for compressive sensing signal reconstruction', IEEE Trans. Inf. Theory, 2009, 55, (5), pp. 2230-2249

19 Baraniuk, R.G., Cevher, V., Duarte, M.F., Hegde, C.: 'Model-based compressive sensing', IEEE Trans. Inf. Theory, 2010, 56, (4), pp. 1982-2001

20 Prokais, J.G.: 'Digital communication' (McGraw-Hill, 1995, 3rd edn.)

21 Baraniuk, R.: 'Compressive sensing', IEEE Signal Process. Mag., 2007, 24, (4), pp. 118-121

22 Paulraj, A.J., Gore, D.A., Nabar, R.U., Bolcskei, H.: 'An overview of MIMO communications - a key to gigabit wireless', Proc. IEEE, 2004, 92, (2), pp. 198-218

23 Bingham, J.A.C.: 'Multicarrier modulation for data transmission: an idea whose time has come', IEEE Commun. Mag., 1999, 28, (5), pp. 5-10

24 Bolcskei, H.: 'MIMO-OFDM wireless systems: basics, perspectives, and challenges', IEEE Wirel. Commun., 2006, 13, (4), pp. 31-37

25 Adeane, J., Rodrigues, M.R.D., Wassell, I.J.: 'Lattice-reduction-aided detection for MIMO-OFDM-CDM communication systems', IET Commun., 2007, 1, (3), pp. 526-531

26 Romberg, J.: 'Imaging via compressive sampling', IEEE Signal Process. Mag., 2008, 25, (2), pp. 14-20

27 Donoho, D.L.: 'Compressed sensing', IEEE Trans. Inf. Theory, 2006, 52, pp. 1289-1306

28 Gilbert, M., Strauss, J., Tropp, A., Vershynin, R.: 'Sublinear approximation of signals', available online, website: http://www.math. lsa.umich.edu/, April 2010

29 Kay, S.M.: 'Fundamentals of statistical signal processing: detection theory' (Prentice-Hall PTR), 1998, vol. 2, pp. 60-120

30 DeVore, M.D.: 'Estimates of error probability for complex gaussian channels with generalized likelihood ratio detection', IEEE Trans. Pattern Anal. Mach. Intell., 2005, 27, (10), pp. 1580-1591 